\title{
The socioeconomic status of childhood cancer survivors and their families determines the extent of the financial burden associated with medical costs
}

\author{
Wonjeong Chae ${ }^{1}$, Juyeong Kim², Sohee Park ${ }^{3}$, Eun-Cheol Park ${ }^{1}$, and Sung-In Jang ${ }^{1}$ \\ ${ }^{1}$ Yonsei University College of Medicine \\ ${ }^{2}$ Sahmyook University \\ ${ }^{3}$ Yonsei University
}

May 20, 2020

\begin{abstract}
Background: The number of cancer survivors, including childhood cancer survivors, is increasing as a consequence of improved therapeutic options. However, the medical costs associated with therapy are high and many families are suffering from the resultant financial burden. Objective: Our study aims to determine the total medical cost for 5 years after the initial diagnosis of childhood cancers. Methods: A customized dataset from the Korean National Health Insurance Claims Database was requested for this study. A total of 7,317 patients were selected to determine the total medical cost related to their treatment. The costs are presented as the $2 \%$ trimmed mean value to exclude extreme costs. The medical costs were further classified according to cancer type, treatment phase, and socioeconomic status. Multiple linear regression analyses were performed. Results: The average total medical cost per patient is 36.8 million Korean Wons (KRW) or 30,665 United States Dollars (USD). Among childhood cancers, leukemia was associated with the highest cost at 53.5 million KRW (USD 44,556). Analysis of socioeconomic status revealed that the higher income group demonstrated higher medical expenditure when compared to other groups. Analysis of the treatment phase showed that costs associated with the early phase of treatment are the highest, especially in the first 3 months after initial diagnosis. Conclusion: Cancer treatments are expensive and are a source of significant financial stress to patients' families. To alleviate the financial burden and reduce the socioeconomic disparities associated with medical care and costs, a better understanding of the current experience of patients and their families is required.
\end{abstract}

\section{Introduction}

Cancer is the second leading cause of death globally. According to a World Health Organization report, cancer resulted in 9.6 million deaths in 2018[1]. In high-income countries, cancer is the number one cause of disease-related death $[2,3]$. Several years of research have improved treatments options for cancer patients, thus improving their survival rate [4,5]. However, the cost of these treatments and the financial burden on patients and their families are high [6-9].

High healthcare expenses are also associated with treatments for childhood cancers. Childhood cancers are a leading cause of death and their prevalence has been increasing since $1980[3,10,11]$. The reported number of childhood cancer cases in Korea was 13,775 in 2014, which was $3.1 \%$ higher than that in 2010 [12]. In the United States, the 5-year survival rate for childhood cancer in 2020 is $84 \%$, whereas it was $58 \%$ in the mid-1970s and $79 \%$ in the mid-1990s [13]. In the United Kingdom, the 5-year survival rate in 2020 is between $76 \%$ and $82 \%$, whereas it was $36 \%$ in 1970 [14]. Along with the increased number of childhood cancer cases and survivors, the medical expense associated with childhood cancer treatment and care has also increased to 87.7 billion Korean Wons (KRW) in 2014 from KRW 83.1 billion in 2010 [15]. The cost of childhood cancer treatment is also high in the United States and begins at 833,000 United States Dollars (USD) [8]. 
Although deaths from childhood cancers are still high, it is curable with advanced medical therapy and technology. Therefore, it is important to provide these medical options to patients, while lowering the associated financial burden on the patients and their families [6,16-18]. For childhood cancer patients, the financial burden on their family is higher than that associated with treatment for adult cancer patients $[9,16,17]$. A study by Merrill et al. reported that the average cost per day for childhood cancer treatments was 700 USD higher than that of adult cancer treatments [19]. Additionally, the hospitalization cost for leukemia patients was higher in children at 55,700 USD compared with adults at 40,200 USD [20].

Due to the high medical costs associated with cancer treatments, the government has established a financial support program for patients. However, the financial burden on families continues to remain high [6-9,1618,21,22]. Importantly, families of childhood cancer patients often experience disruptions in the regular employment of the caregiver, which significantly diminishes the household income [23-25]. Some studies show that families with childhood cancer patients are driven below the poverty line due to high medical costs $[18,23,25,26]$. Furthermore, some patients and their families make treatment decisions based on the associated cost, which can lead to undesirable outcomes [27]. Therefore, it is important to prioritize and allocate the limited healthcare resources in an efficient manner and provide financial support to patients and their families.

Although understanding the economic impact of childhood cancer is essential, there are very few studies describing the financial burden associated with medical treatments and its impact on patients' families [22, 24]. The purpose of this study is to investigate the burden of childhood cancer costs on cancer survivors and their families based on socioeconomic status. To this end, we examined the medical costs associated with cancer survival for 5 years after the initial cancer diagnosis, based on childhood cancer type and socioeconomic status.

\section{Materials and Methods}

\section{Data}

The National Health Insurance Service (NHIS) is a mandatory health insurance system in Korea and their database includes $97 \%$ of the Korean population. The NHIS database includes medical diagnoses, treatments, drug information, hospital information, and demographic information. Medical diagnoses are indicated using International Statistical Classification of Diseases and Related Health Problems, 10th revision (ICD-10) codes. For this study, we requested a customized dataset of children who were diagnosed with cancer (C00C99) and their parents' income data from 2002 to 2015 from the Korean National Health Insurance Claims Database.

\section{Study population}

From a total of 21,205 patients aged 0-17 who were diagnosed with cancer, we applied a 1-year washout period to identify and include newly diagnosed cancer patients for the study. To obtain socioeconomic status, we extrapolated insurance payment data from the patient's parent income data and linked the two parameters together. We excluded 5,598 patients who lacked parent data. Our study design also excluded cancer patients that died within 5 years of diagnosis. Finally, we excluded 150 patients whose medical expenditure was in the upper and lower $1 \%$ of all medical costs. A total of 7,317 patients were ultimately used for the study.

\section{Medical Cost}

Medical expenses in our study are reported from the insurer's perspective and are presented as average costs (mean values). To eliminate the extreme values, we applied a $2 \%$ trimmed mean and excluded the upper and lower $1 \%$ values. The cost includes all medical treatment expenses for 5 years after the initial diagnosis. For costs between the years 2003 to 2014, we applied an adjustment in the unit price per score to reflect the actual value of the cost in the year 2015. The medical costs were corrected by multiplying the unit price by the following adjustment rates: 1.30 (2003), 1.26 (2004), 1.23 (2005), 1.19 (2006), 1.15 (2007), 1.13 (2008), 
1.10 (2009), 1.09 (2010), 1.08 (2011), 1.06 (2012), 1.04 (2013), and 1.02 (2014). In this study, all amounts are presented in KRW [1 USD is equivalent to 1,200 KRW in average].

\section{Cancer types}

In our study, we grouped childhood cancers into six categories based on the National Cancer Registry Annual Report: leukemia (C91-C95), brain and central nervous system cancer (C70-C72), non-Hodgkin's lymphoma (C82-C85, C96), bone and articular cartilage cancer (C40-C41), mesothelioma soft tissues (C45-C49), and the remaining group of 'other' cancers.

We classified cancer treatment into 3 phases: early, middle, and late phases. The early phase is the first year following diagnosis, the middle phase includes the second to the fourth year following diagnosis, and the late phase is the fifth year following diagnosis. The early phase has been further divided into $3,6,9$, and 12 months.

\section{Socioeconomic Status}

Socioeconomic status is classified based on the parents' health insurance premium, which is reflective of the household income. The status is categorized into the following quartile ranges: low (KRW 0-43,260,000), midlow (KRW 43,261,000-74,555,000), mid-high (KRW 75,556,000-122,370,000), and high (KRW 122,371,000 or more) .

\section{Statistical Analysis}

The main analysis was performed using multivariable linear regression. The analysis was performed to determine the association between the outcome variable, cost of cancer treatments, and covariates. For subgroup analysis, we performed multivariable linear regressions based on the patients' socioeconomic status. All analyses were performed using the SAS software, version 9.4 (SAS Institute, Cary, North Carolina, USA).

\section{Results}

Table 1 shows the general characteristics of the study population. In our study, we included 7,317 patients, of whom $27.8 \%$ were leukemia patients, $15.2 \%$ were brain and central nervous system cancer patients, $9.2 \%$ were non-Hodgkin's lymphoma patients, $7.0 \%$ were bone and articular cartilage cancer patients, $5.1 \%$ were mesothelioma soft tissue cancer patients, and $35.8 \%$ were 'other' cancer patients.

Table 2 displays the average medical cost incurred by childhood cancer survivors from the time of initial diagnosis until the end of the next 5 years. The total medical cost per patient is 36.8 million KRW (USD 30,665). Inpatient services cost 28.3 million KRW (USD 23,600), whereas outpatient services cost 8 million KRW (USD 7,078). When classified by cancer type, leukemia was associated with the highest cost at 53.5 million KRW (USD 44,556) followed by bone and articular cartilage cancer at 52.0 million KRW (USD 43,374) (Supplementary table 1).

Results of a regression analysis on total medical costs for childhood cancer survivors are presented in Table 3. Patients undergoing chemotherapy, radiotherapy, and surgery bore higher medical costs compared with those who did not receive major treatments. When analyzed according to socioeconomic status, the medical cost was greater in patients of higher socioeconomic status. This result was unaffected by cancer type (Supplementary table 2). To investigate the duration of services received, regression analyses on the number of days of medical service use was performed (Table 4). Leukemia patients used medical services for a longer duration (104.0 days) compared to any other childhood cancer patients in both inpatient (67.4 days) and outpatient (36.6 days) service categories.

Figure 1 demonstrates the medical costs borne by childhood cancer survivors, based on their socioeconomic status, in each treatment phase and the cumulative medical cost. Medical costs for patients were high in the early phase of treatment. When analyzed according to socioeconomic status, increased medical costs were observed in the first 3 months following diagnosis for the high status category. The medical costs based on socioeconomic status are as follows: low status at 11.7 million KRW (USD 9,776); mid-low at 12.1 
million KRW (USD 10,116); mid-high at 12.2 million KRW (USD 11,083); and high at 15.0 million KRW (USD 12,508) for the same treatment phase. The cumulative costs classified according to cancer types are presented in Supplementary table 3.

\section{Discussion}

Our study focused on the medical treatment-associated financial burden borne by childhood cancer patients and their families based on their socioeconomic status. Childhood cancer impacts not only the patient, but also the family. In our study, we found that medical expenditure varied based on socioeconomic status and treatment phase. Previous studies on the financial burden of childhood cancers were limited to specific hospitals or areas with small sample sizes. Because our study utilized NHIS data, our sample size is greater and more representative of the Korean population compared to previous studies.

We found that the medical expenditure at early phases of treatment is the highest. Clinical research has reported that during the early phases of cancer treatment, intensive care, lengthy hospitalization, and frequent outpatient services are needed. Therefore, after cancer diagnosis, patients and their families experience financial stress in addition to the psychological stress associated with the diagnosis itself $[6,9,24,26]$. Similar to our results, Warner et al. reported that in the first year of diagnosis, childhood cancer patients and families are financially vulnerable [27]. Considering that the main caregiver for the patient is a parent, the inevitable disruption of parents' employment in many cases increases the financial burden on the family $[7,22,26]$. Although this financial burden affects families independently of socioeconomic status, patients and their families in the low status category suffer more [18,26,28-31].

Cancer treatment requires long-term care that can place an immense financial burden on patients' families. Some patients could make treatment decisions based on costs [16,29-31]. To reduce this financial burden, the Korean government has a special system in place wherein a cancer patient pays $5 \%$ of the total out-ofpocket medical costs covered by insurance after the diagnosis [32]. While this system provides some relief to patients and families, the treatments are expensive, and some facets of treatment are not covered by the national health insurance. As our results demonstrate, the medical cost for childhood cancer survivors varies based on socioeconomic status. Importantly, the results only include treatments covered by insurance. If we factored in non-covered treatments in our calculations, it would further exacerbate the gap in financial burden based on socioeconomic status. In addition to the special system described above, the government also provides financial support to low-income patients who pay health insurance premiums below KRW 100,000 [32]. However, our study found that medical costs of the low status group and the mid-low status group showed no significant difference. In fact, the mid-low status group had decreased cumulative medical costs at 1 year post-diagnosis. Therefore, an effort to expand financial support for families is required.

In conclusion, as improved medical treatments increase the numbers of childhood cancer survivors, the economic burden associated with medical treatment also increases. A better understanding of how the financial burden is unevenly distributed across socioeconomic classes is required to reduce the disparities in medical treatment received by patients. In addition, considering the duration of healthcare needed for patients, establishing a continuous economic support policy would be beneficial for lowering the financial burden on childhood cancer patients and their families.

\section{Limitations}

There are some limitations to our study. First, the medical expenses calculated in our study only indicate those covered by health insurance. We were unable to obtain non-covered medical expenses; however, most costs related to childhood cancers are covered by national health insurance. Secondly, we did not include the medical expenses associated with subsequent non-cancer-related doctor visits and treatments. However, as cancer treatments comprise very intensive care, we expect that other treatment costs would not substantially alter our results. Thirdly, we only considered whether the patients received treatments such as surgery, chemotherapy, and radiation, but not the frequency at which they received them. Therefore, further studies are necessary to overcome these limitations. 


\section{References}

1. World Health Organization. Cancer.https://www.who.int/news-room/fact-sheets/detail/cancer. Accessed December 42019.

2. Dagenais GR, Leong DP, Rangarajan S, Lanas F, Lopez-Jaramillo P, Gupta R, Diaz R, Avezum A, Oliveira GBF, Wielgosz A, Parambath SR, Mony P, Alhabib KF, Temizhan A, Ismail N, Chifamba J, Yeates K, Khatib R, Rahman O, Zatonska K, Kazmi K, Wei L, Zhu J, Rosengren A, Vijayakumar K, Kaur M, Mohan V, Yusufali A, Kelishadi R, Teo KK, Joseph P, Yusuf S. Variations in common diseases, hospital admissions, and deaths in middle-aged adults in 21 countries from five continents (PURE): a prospective cohort study. Lancet 2020:395:785-794.

3. World Health Organization. Cancer in Children.https://www.who.int/news-room/factsheets/detail/cancer-in-children. Accessed December 42019.

4. Ward E, DeSantis C, Robbins A, Kohler B, Jemal A. Childhood and adolescent cancer statistics, 2014. CA Cancer J Clin 2014:64:83-103.

5. Linabery AM, Ross JA. Childhood and adolescent cancer survival in the US by race and ethnicity for the diagnostic period 1975-1999. Cancer 2008:113:2575-2596.

6. Luengo-Fernandez R, Leal J, Gray A, Sullivan R. Economic burden of cancer across the European Union: a population-based cost analysis. Lancet Oncol 2013:14:1165-1174.

7. Guy GP, Yabroff KR, Ekwueme DU, Smith AW, Dowling EC, Rechis R, Nutt S, Richardson LC. Estimating the health and economic burden of cancer among those diagnosed as adolescents and young adults. Health affairs (Project Hope) 2014:33:1024-1031.

8. National Children's Cancer Society. The Economic Impact of Childhood Cancer. St. Louis, Missouri2018.

9. Sneha LM, Sai J, Ashwini S, Ramaswamy S, Rajan M, Scott JX. Financial Burden Faced by Families due to Out-of-pocket Expenses during the Treatment of their Cancer Children: An Indian Perspective. Indian J Med Paediatr Oncol 2017:38:4-9.

10. Kellie SJ, Howard SC. Global child health priorities: what role for paediatric oncologists? Eur J Cancer 2008:44:2388-2396.

11. Steliarova-Foucher E, Colombet M, Ries LA, Moreno F, Dolya A, Bray F, Hesseling P, Shin HY, Stiller CA, Bouzbid S. International incidence of childhood cancer, 2001-10: a population-based registry study. The Lancet Oncology 2017:18:719-731.

12. Ministry of Health \& Welfare. Larger proportion of leukemia found among childhood cancer patients than adults cancer patientshttp://www.korea.kr/news/pressReleaseView.do?newsId=1560369058pageIndex=49286repCodeType= 02-2983endDate=2019-09-308srch Word $=$.

13. American Cancer Society. Cancer Facts \& Figures 2020. Atlanta: American Cancer Society; 2020.

14. Cancer Research UK. Children's cancers survival statistics.https://www.cancerresearchuk.org/healthprofessional/cancer-statistics/childrens-cancers/survival\#heading-Zero. Accessed December 122019.

15. Ministry of Health \& Welfare. $152.1 \%$ of increase in medical expences for childhood cancer treatment during recent 5

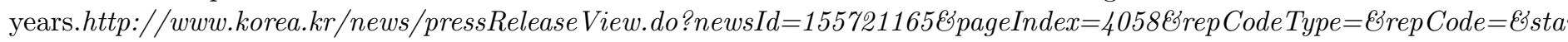
02-2982019-04-2483srchWord=. Accessed December 72019.

16. Eiser C, Upton P. Costs of caring for a child with cancer: a questionnaire survey. Child: Care, Health and Development 2007:33:455-459.

17. Tsimicalis A, Stevens B, Ungar WJ, McKeever P, Greenberg M. The cost of childhood cancer from the family's perspective: a critical review. Pediatr Blood Cancer 2011:56:707-717. 
18. Warner EL, Kirchhoff AC, Nam GE, Fluchel M. Financial Burden of Pediatric Cancer for Patients and Their Families. J Oncol Pract 2015:11:12-18.

19. Chaya T Merrill, Mika Nagamine, Hambrick MM. Pediatric Hospital Stays for Cancer, 2005 Statistical Brief \#37.https://www.ncbi.nlm.nih.gov/books/NBK61974/. Accessed December 202019.

20. Chaya T Merrill, Carol Stocks, Stranges E. Trends in Uninsured Hospital Stays, 1997-2006 Statistical Brief \#67.https://www.ncbi.nlm.nih.gov/books/NBK54561/. Accessed Decmber 122019.

21. Wang J, Dong Z, Hong SH, Suda KJ. A comparison of direct medical costs across racial and ethnic groups among children with cancer. Current medical research and opinion 2008:24:847-858.

22. De Oliveira C, Bremner KE, Liu N, Greenberg ML, Nathan PC, McBride ML, Krahn MD. Costs for Childhood and Adolescent Cancer, 90 Days Prediagnosis and 1 Year Postdiagnosis: A Population-Based Study in Ontario, Canada. Value Health 2017:20:345-356.

23. Bona K, Dussel V, Orellana L, Kang T, Geyer R, Feudtner C, Wolfe J. Economic impact of advanced pediatric cancer on families. Journal of pain and symptom management 2014:47:594-603.

24. Dussel V, Bona K, Heath JA, Hilden JM, Weeks JC, Wolfe J. Unmeasured costs of a child's death: perceived financial burden, work disruptions, and economic coping strategies used by American and Australian families who lost children to cancer. Journal of clinical oncology 2011:29:1007.

25. Limburg H, Shaw AK, McBride ML. Impact of childhood cancer on parental employment and sources of income: a Canadian pilot study. Pediatric blood \& cancer 2008:51:93-98.

26. Mader L, Roser K, Baenziger J, Tinner EM, Scheinemann K, Kuehni CE, Michel G. Household income and risk-of-poverty of parents of long-term childhood cancer survivors. Pediatr Blood Cancer 2017:64.

27. Warner EL, Kirchhoff AC, Nam GE, Fluchel M. Financial Burden of Pediatric Cancer for Patients and Their Families. Journal of oncology practice 2015:11:12-18.

28. Guy GP, Berkowitz Z, Ekwueme DU, Rim SH, Yabroff KR. Annual economic burden of productivity losses among adult survivors of childhood cancers. Pediatrics 2016:138:S15-S21.

29. Dreyer MS, Nattinger AB, McGinley EL, Pezzin LE. Socioeconomic status and breast cancer treatment. Breast Cancer Res Treat 2018:167:1-8.

30. Singh GK, Jemal A. Socioeconomic and Racial/Ethnic Disparities in Cancer Mortality, Incidence, and Survival in the United States, 1950-2014: Over Six Decades of Changing Patterns and Widening Inequalities. Journal of environmental and public health 2017:2017:2819372-2819372.

31. Wong SL, Gu N, Banerjee M, Birkmeyer JD, Birkmeyer NJ. The impact of socioeconomic status on cancer care and survival. Journal of Clinical Oncology 2011:29:6004-6004.

32. Choi JW, Kim J-H, Park E-C. The Relief Effect of Copayment Decreasing Policy on Unmet Needs in Targeted Diseases. Health Policy and Management 2014:24.

Figure title : Medical cost childhood cancer survivor by socioeconomic status in each treatment phase cost and cumulative cost

Figure legend: +Presented in KRW (KRW 1,200)

\section{Hosted file}

0510_table all.docx available at https://authorea.com/users/324437/articles/452632-thesocioeconomic-status-of-childhood-cancer-survivors-and-their-families-determines-theextent-of-the-financial-burden-associated-with-medical-costs 


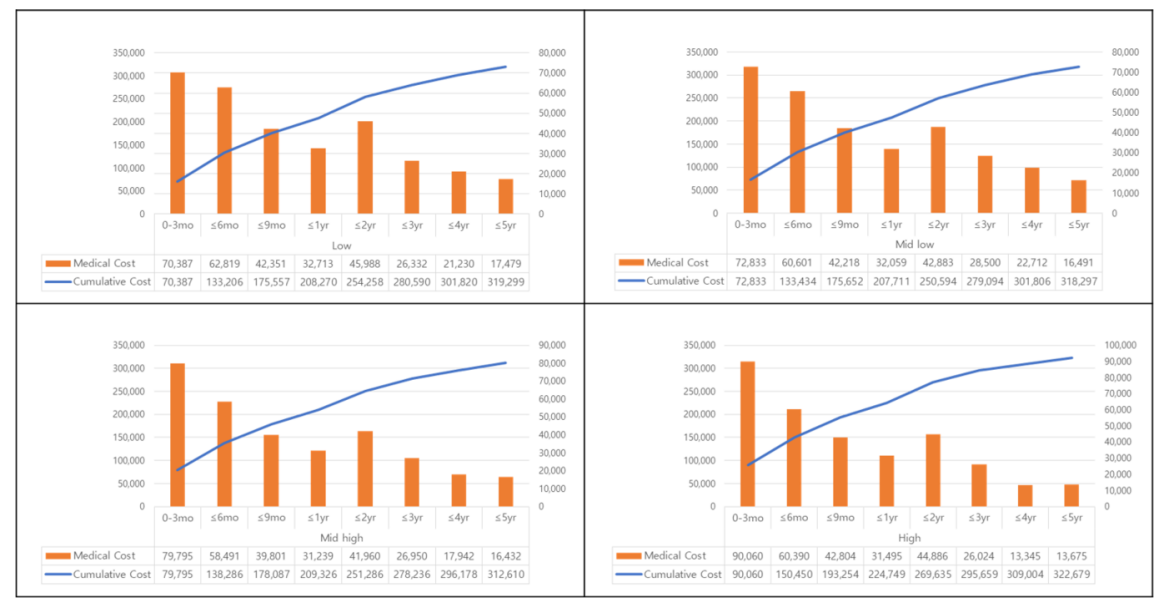

